\title{
A pilot study of the ultrathin cryoprobe in the diagnosis of peripheral pulmonary ground-glass opacity lesions
}

\author{
Simin Jiang ${ }^{1,2,3 \#}$, Xiaojun Liu ${ }^{1,2,4 \#}$, Junxiang Chen ${ }^{1,2,3}$, Haifeng Ma $^{5}$, Fangfang Xie ${ }^{1,2,3}$, Jiayuan Sun ${ }^{1,2,3}$ \\ ${ }^{1}$ Department of Respiratory Endoscopy, ${ }^{2}$ Department of Respiratory and Critical Care Medicine, Shanghai Chest Hospital, Shanghai Jiao Tong \\ University, Shanghai, China; ${ }^{3}$ Shanghai Engineering Research Center of Respiratory Endoscopy, Shanghai, China; ${ }^{4}$ Department of Respiratory \\ and Critical Care Medicine, First Hospital of Shanxi Medical University, Taiyuan, China; ${ }^{5}$ Institute of Respiratory Disease, First Hospital of China \\ Medical University, Shenyang, China \\ Contributions: (I) Conception and design: J Sun; (II) Administrative support: None; (III) Provision of study materials or patients: J Sun; (IV) Collection \\ and assembly of data: X Liu, J Chen, S Jiang, H Ma, F Xie, J Sun; (V) Data analysis and interpretation: X Liu, J Chen, S Jiang, H Ma, F Xie, J Sun; (VI) \\ Manuscript writing: All authors; (VII) Final approval of manuscript: All authors. \\ \#These authors contributed equally to this work. \\ Correspondence to: Jiayuan Sun. Department of Respiratory Endoscopy and Department of Respiratory and Critical Care Medicine, Shanghai Chest \\ Hospital, Shanghai Jiao Tong University, 241 West Huaihai Road, Shanghai 200030, China. Email: xkyyjysun@163.com.
}

Background: It is very difficult to obtain samples of peripheral pulmonary ground-glass opacity lesions (GGOs) by traditional transbronchial biopsy. This study was conducted to evaluate the diagnostic efficacy and safety of transbronchial cryobiopsy (TBCB) of GGOs using a newly developed ultrathin cryoprobe with an outer diameter of $1.1 \mathrm{~mm}$.

Methods: We retrospectively analyzed 20 patients with 23 GGOs who underwent TBCB using the ultrathin cryoprobe from October 2018 to November 2019 in the Shanghai Chest Hospital. The TBCB procedure was performed under the guidance of virtual bronchoscopic navigation (VBN), electromagnetic navigation bronchoscopy (ENB), endobronchial ultrasound, and fluoroscopy. We collected the baseline information of participants, reported diagnostic yield and complications, and analyzed factors may have affected the diagnostic yield.

Results: A total of 23 GGOs (12 pure GGOs, 11 mixed GGOs), with an average diameter of 21.58 $11.88 \mathrm{~mm}$, underwent TBCB, and the diagnostic yield was 82.61\% (19/23). Of the 19 GGOs diagnosed by TBCB, 12 were adenocarcinomas, 5 were inflammation, 1 was occupational interstitial lung disease, and 1 was a pulmonary meningothelial-like nodule. The remaining 4 undiagnosed lesions were confirmed to be adenocarcinomas by further analysis. The diagnostic yield was unchanged by factors including size (GGOs $\geq 20 \mathrm{~mm}$, GGOs $<20 \mathrm{~mm}$ ), navigation (VBN, ENB), fluoroscopic visibility (visible, invisible), GGOcomponent (pure GGOs, mixed GGOs), and guide sheath (K-201, K203). There was no presentation of pneumothorax or severe hemorrhage.

Conclusions: The ultrathin cryoprobe is feasible, safe, and has a high diagnostic yield in the diagnosis of pulmonary GGOs. There is vast potential for the ultrathin cryoprobe as a tool for the diagnosis of GGOs, especially for cases suspicious of early-stage lung cancer.

Trial registration: ClinicalTrials.gov. No: NCT03716284. Registered: 20 October, 2018. URL: ClinicalTrials.gov.

Keywords: Diagnosis; ground-glass opacity (GGO); transbronchial cryobiopsy (TBCB); transbronchial lung biopsy (TBLB); lung cancer

Submitted Aug 04, 2020. Accepted for publication Sep 24, 2020.

doi: $10.21037 /$ tlcr-20-957

View this article at: http://dx.doi.org/10.21037/tlcr-20-957 


\section{Introduction}

Ground-glass opacity lesions (GGOs) are characterized as an elevation of lung opacity on high-resolution computed tomography (HRCT) without obscurement of the bronchi and vessels (1). Any changes that induce partial airspace filling and thickening of the interlobular septa will lead to GGOs, as seen with interstitial pneumonia, inflammation, and preinvasive and invasive carcinoma $(2,3)$. Classification of GGOs into pure and mixed types is made according to whether there is involvement of solid components (4). Pulmonary GGOs require unique consideration apart from solid lesions, because they are more likely to be malignant yet paradoxically indicate a better prognosis (5). The unique structures of pulmonary GGOs have also challenged the effectiveness of traditional non-surgical diagnostic tools that have been widely used for solid lesions.

Transthoracic needle aspiration (TTNA), despite having a diagnostic sensitivity of $90 \%$ for lung cancer (6), may not be very efficient in the diagnosis of GGOs. Apart from the multiple complications induced by TTNA (7), problems are posed by the small size of GGOs, that they appear to change position with respiratory motion, and their loose structures are easily obscured by bleeding; all of these features increase the difficulty of a second biopsy. Advanced bronchoscopy, including endobronchial ultrasound (EBUS), virtual bronchoscopic navigation (VBN), and guide sheath (GS), have been conducted to improve the diagnostic yield of GGOs (8-11). Cryobiopsy, which has been commonly used to identify interstitial pulmonary diseases and endobronchial tumors, presented unique value in collecting large and well-preserved specimens, thus expanding its diagnostic scope to peripheral pulmonary lesions (PPLs) $(12-15)$. But until now, study has not been conducted to explore the value of transbronchial cryobiopsy (TBCB) for diagnosing peripheral pulmonary GGOs.

We innovatively used an ultrathin cryoprobe, with an outer diameter of $1.1 \mathrm{~mm}$, incorporated with $\mathrm{VBN}$ or electromagnetic navigation bronchoscopy (ENB), as well as EBUS-GS and X-ray fluoroscopy to diagnose peripheral pulmonary GGOs. As far as we know, this was the first time the ultrathin cryoprobe has been clinically applied for this diagnostic purpose. We present the following article in accordance with the STARD reporting checklist (available at http://dx.doi.org/10.21037/tlcr-20-957).

\section{Methods}

\section{Participants}

From October 2018 to November 2019, 20 consecutive patients with 23 GGOs that underwent TBCB by the ultrathin cryoprobe in the Shanghai Chest Hospital were prospectively recorded and retrospectively analyzed. Inclusion criteria: (I) patients >18 years-old with GGOs suspicious of malignancy. (II) The GGOs were located at inferior segmental bronchus. Exclusion criteria: (I) GGOs with endobronchial concomitant. (II) Patients with contraindications to bronchoscopy, such as bleeding diathesis, anticoagulation treatment, severe cardiopulmonary dysfunction, pregnancy, etc. (III) Patients' refusal of this intervention. The protocol was approved by the local ethics committee of the Shanghai Chest Hospital (KS1829). All patients provided written informed consent. All procedures performed in this study involving human participants were in accordance with the Declaration of Helsinki.

Each patient underwent HRCT before bronchoscopy to evaluate lesion size, lobar location, bronchus sign, nodule numbers, and the proportion of GGO component. Lesion size was defined as the longest diameter on the largest section of HRCT. The ratio of GGO component refers to the diameter of a GGO element attenuated at the mediastinal window compared to the diameter of the lesion at the lung window. Bronchus sign describes the positional relationship of the lesion and nearby bronchus on HRCT, and a lesion with a bronchus directly leading into it is defined as a positive bronchus sign.

Patients underwent bronchoscopy with general anesthesia. Scanning data of the HRCT were transferred into a LungCare navigation system (LungCare Medical Technologies Ltd., Inc., Suzhou, China) to construct virtual image and determine the biopsy route before insertion of the bronchoscope. We routinely applied VBN and ENB in accordance with the previously reported instructions (16). We adopted 3 kinds of modalities, as shown in Figure 1. The standard bronchoscope (BF-1T260 or BF-1TQ290, Olympus, Tokyo, Japan) was incorporated with a K-203 GS (Olympus) and an UM-S20-20R probe (Olympus). The thin bronchoscope (BF-P290 or BF-P260F, Olympus), was incorporated with a K-201 GS (Olympus) and an UM-S20$17 \mathrm{~S}$ probe (Olympus). The ultrathin bronchoscopy (UTB, 


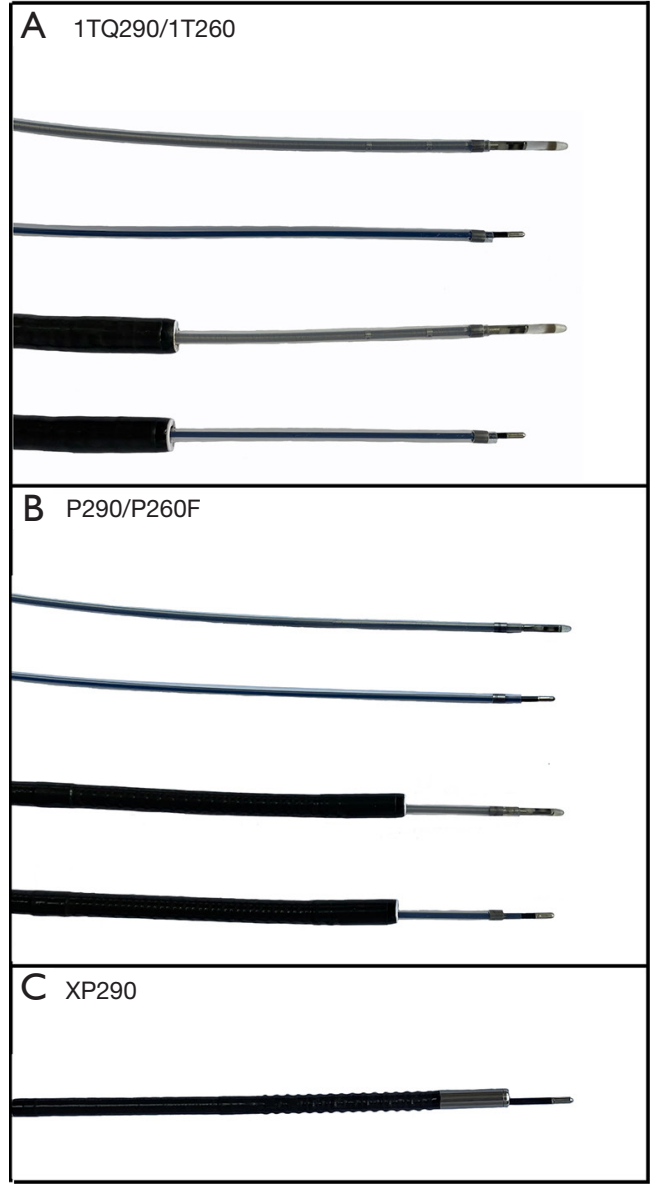

Figure 1 Different assembly of bronchoscope, endobronchial ultrasound (EBUS), guide sheath (GS), and ultrathin cryoprobe. (A) The standard bronchoscope (BF-1TQ290/BF-1T260, distal end outer diameter $5.9 \mathrm{~mm}$, working channel $3.0 \mathrm{~mm}$ ), EBUS (UMS20-20R, outer diameter $1.7 \mathrm{~mm}$ ), GS (K-203, outer diameter $2.55 \mathrm{~mm}$ ), and the ultrathin cryoprobe (outer diameter $1.1 \mathrm{~mm}$ ) were used together. (B) The thin bronchoscope (BF-P290/BFP260, distal end outer diameter $4.2 \mathrm{~mm}$, working channel $2.0 \mathrm{~mm}$ ) was engaged with EBUS (UM-S20-17S, outer diameter $1.4 \mathrm{~mm}$ ), GS (K-201, outer diameter $1.95 \mathrm{~mm}$ ), and the ultrathin cryoprobe. (C) The ultrathin bronchoscope (UTB, BF-XP290, Olympus, distal end outer diameter $3.1 \mathrm{~mm}$, working channel $1.2 \mathrm{~mm}$ ) was directly combined with the ultrathin cryoprobe.

BF-XP290, Olympus) was performed without EBUS or GS. Navigation (VBN or ENB) and bronchoscopes were applied according to individual judgment of the operators. Once the bronchoscope reached the targeted bronchus, the combined ultrasound probe and GS were inserted, and their position and orientation were adjusted under the fluoroscope, until
EBUS images of the GGOs were able to be obtained.

The ultrasound signal was recorded as "Blizzard sign" and "Mixed blizzard sign". The blizzard sign usually appeared at pure GGOs, as an increase in intensity and radius of the whitish acoustic shadow of the normal lung tissue $(>1 \mathrm{~cm})(17,18)$. The mixed blizzard sign appeared in mixed GGOs as a combination of blizzard sign and a diffuse heterogeneity scattered with hyperechoic dots, linear arcs, and vessels (17). We divided the lesions into 3 groups, "within", "adjacent to", and "invisible", by the relationship between the probe position and the localization of the lesions (19). If the GGO was invisible on the EBUS image, biopsy would be applied under navigation bronchoscopy with fluoroscopic guidance.

The ultrasound probe would be withdrawn after localizing the targeted lesions, then a $1.1 \mathrm{~mm}$ cryoprobe (Erbe, Tuebingen, Germany) was inserted, and after freezing for 3-5 s, it was removed with a specimen inside it. The cryoprobe was small enough to be removed from the working channel of the thick GS (K-203), leaving the standard bronchoscope and GS in situ. However, for a thin bronchoscope, the cryoprobe would be removed en bloc with the thin GS (K-201) and a specimen. For UTB, the cryoprobe worked alone without EBUS or GS due to its very small working channel; the cryoprobe and the UTB were removed together out of the airway (Figures 2-4). The procedure was repeated several times from the remote to distal ends of the targeted bronchus based on former localization under fluoroscopic guidance, without being rechecked by EBUS. The frozen specimens from each lesion were thawed first in saline at room temperature, next transferred to formalin for fixation, and then collected as one sample; the sample size was recorded. After TBCB, if deemed necessary by the operators, forceps, bronchial brush, and sheath wash were introduced for histological and cytological examination.

The final diagnosis was confirmed by comprehensive analysis of evidence, including histology (surgery, TBCB, forceps), microbiology, and clinical follow-up. The diagnostic yield of TBCB was defined as the number of GGOs correctly diagnosed by cryobiopsy out of the total number of GGOs that received cryobiopsy, which means either a definitive malignancy, or a specific benign result (e.g., granuloma, fibrosis, inflammation, or definitive microbiological evidence), both of which should be consistent with the clinical outcomes of a $>6$-month followup. If the sample was non-specific (e.g., bronchial mucosa and normal lung tissue), it was considered to be unqualified. 

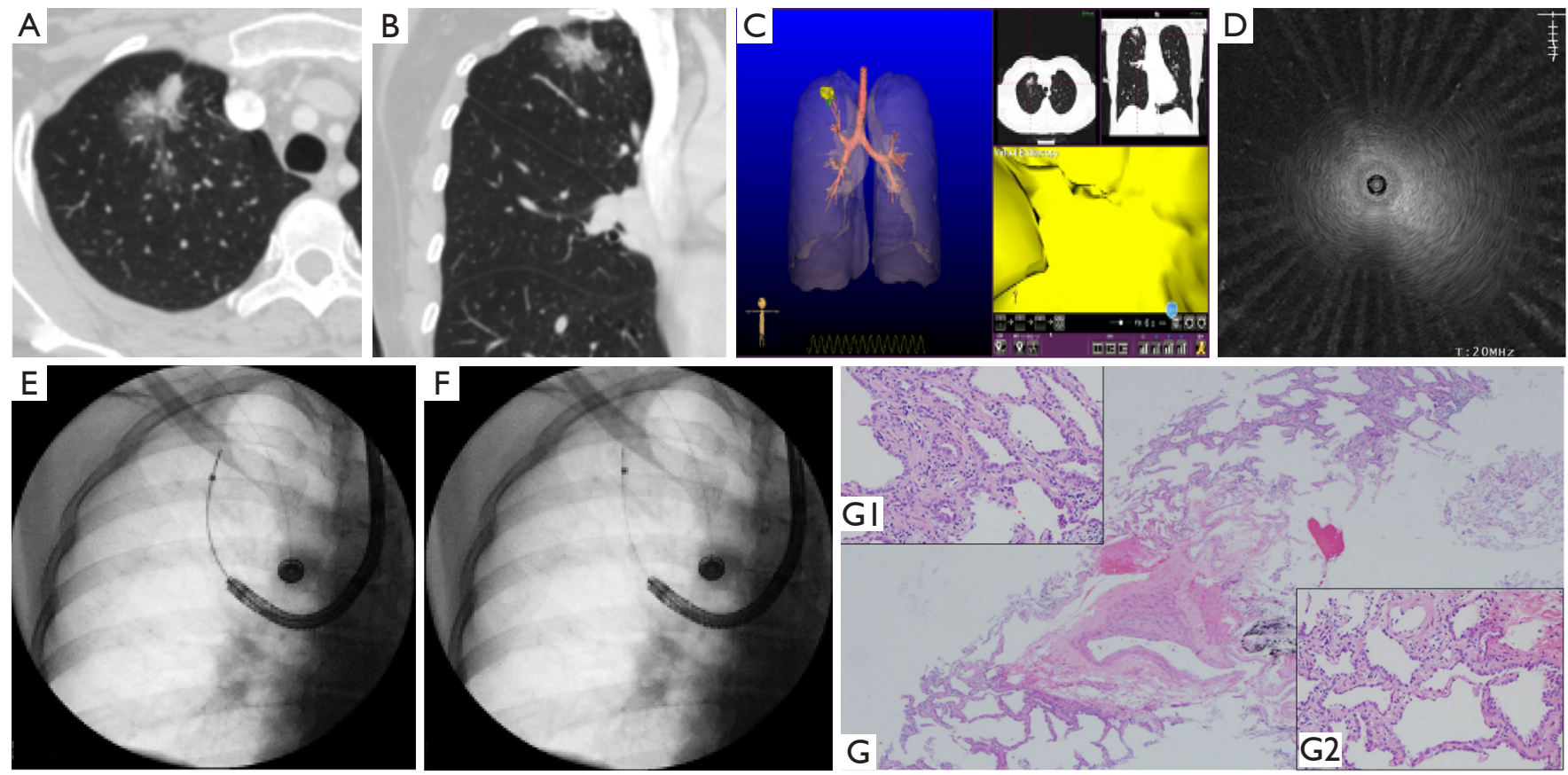

Figure 2 Representative case 1 of (TBCB) using the standard bronchoscope (BF-1TQ290) with electromagnetic navigation bronchoscopy (ENB). (A) The axial section of high-resolution computed tomography (HRCT) showed that the GGO was located in the apical segment of the right upper lobe (RUL). (B) The coronal plane of HRCT showed where the GGO was located. (C) The ENB presented a real-time interface where the tracking wire reached the target. (D) The endobronchial ultrasound (EBUS) image showed the blizzard sign. (E) The location of the EBUS probe (UM-S20-20R) was visualized by X-ray fluoroscopy to confirm its arrival. (F) After confirming arrival of the bronchoscope, the EBUS probe was retracted and cryobiopsy was performed via the K-203 GS. (G) H\&E staining of the specimen with the magnification of $\times 4$. (G1) Magnification of the right upper corner of the specimen showed acinar adenocarcinoma (magnification, $\times 20)$. (G2) Magnification of the left lower corner of the specimen displays adenocarcinoma with lepidic pattern (magnification, $\times 20$ ).

\section{Statistical analysis}

Descriptive statistics were recorded as frequency, percentage, median (range), and mean \pm standard deviation. Factors affecting the diagnostic yield were evaluated by Fisher's exact test. Univariate analysis was adopted to identify the parameters that variated the diagnostic yield of TBCB. All $\mathrm{P}$ values were bilateral. A $\mathrm{P}$ value $<0.05$ was considered statistically significant. The statistical analysis was conducted within SPSS version 20.0 statistical software (IBM, Armonk, NY, USA).

\section{Results}

A total of 20 participants with 23 GGOs (12 pure GGOs and 11 mixed GGOs) received TBCB, of which 3 patients underwent biopsy at 2 sites (Table 1). The average diameter of the GGOs was $21.58 \pm 11.88 \mathrm{~mm}$. All GGOs were found to be bronchus sign positive, of which 21 had ground-glass composition of $>50 \%$, and 14 (10 pure GGOs and 4 mixed GGOs) could not be visualized by fluoroscopy.

We adopted ENB in 9 lesions and VBN in 14 lesions. Except for 1, which underwent TBCB without EBUS, all the other 22 lesions (11 pure GGO and 11 mixed GGO) were identified by EBUS, and all the ultrasound probes were located "within" the GGOs. Of the 11 pure GGOs revealed on EBUS, 10 showed blizzard sign, and 1 showed mixed blizzard sign. The mixed blizzard sign may have resulted from the bronchial lavage with $20 \mathrm{~mL}$ normal saline having been conducted before TBCB in this case, and the residual water in the lesion changed the ultrasound image. Of the 11 mixed GGOs, 8 showed the mixed blizzard sign, and 3 showed the blizzard sign, which was probably due to the probe being positioned in the vicinity of the GGO component.

Each lesion underwent 3 [1-7] cryobiopsies, and we acquired 3 [1-5] pieces of specimen from each. The 

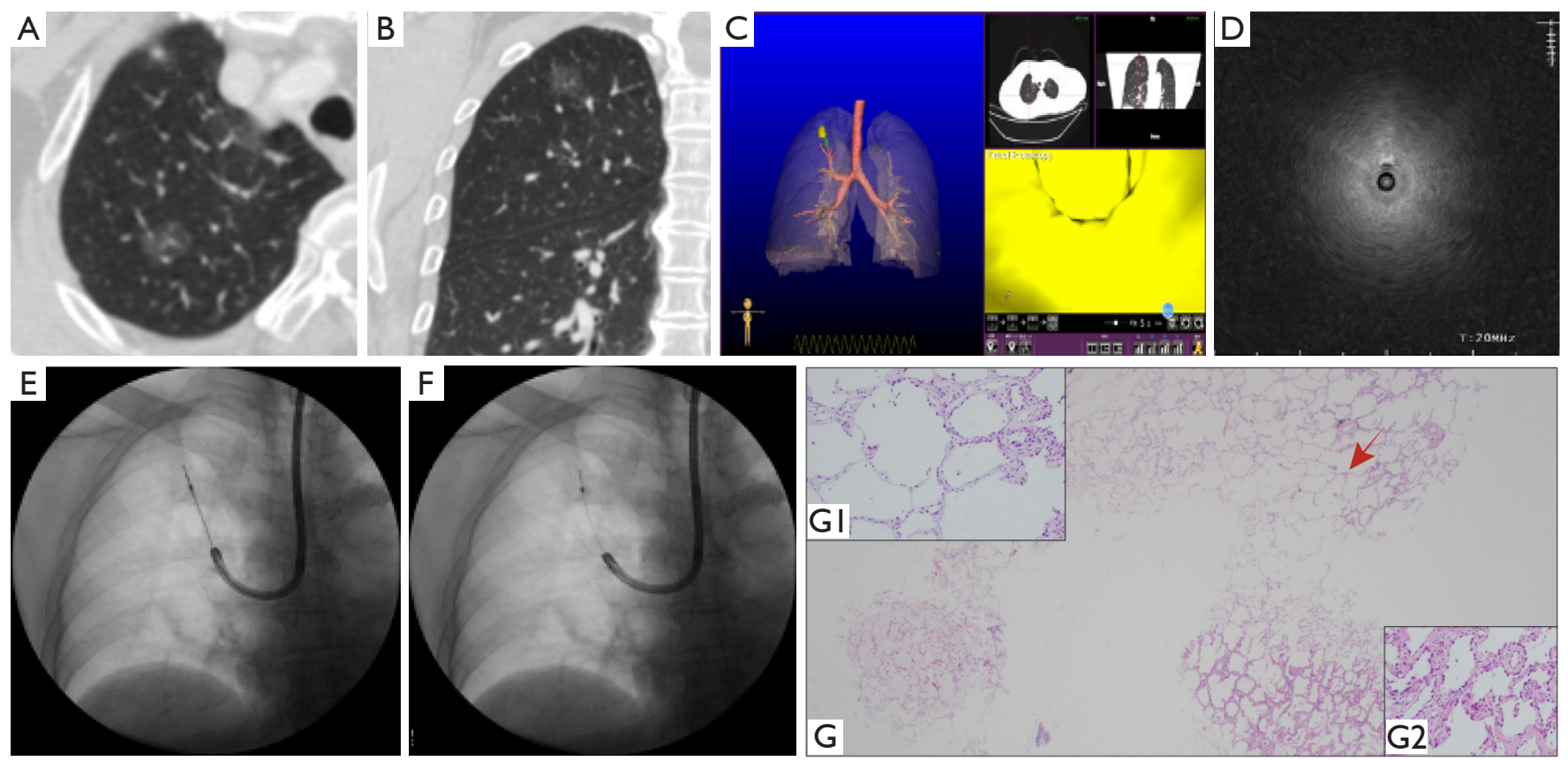

Figure 3 Representative case 2 of transbronchial cryobiopsy (TBCB) using the thin bronchoscope (BF-P290) with ENB. (A) The highresolution computed tomography (HRCT) axial section showed that the ground-glass opacity (GGO) was located in the apical segment of the right upper lung (RUL). (B) The HRCT coronal plane showed where the GGO was located. (C) Electromagnetic navigation bronchoscopy (ENB) showed the real-time interface where the positioning wire reaches the target. (D) Endobronchial ultrasound (EBUS) displayed "Blizzard sign". (E) Location of the ultrasound probe (UM-S20-17S) was visualized by X-ray to confirm its arrival. (F) After confirming the arrival of bronchoscope, the ultrasound probe was retracted and cryobiopsy was performed via the K-201 GS. (G) Hematoxylin and eosin (H\&E) staining of the specimen with the magnification of $\times 2$. (G1) Magnification of the pointed area displays the transition from normal pulmonary alveoli to atypical hyperplastic area (magnification, $\times 10)$. (G2) Magnification of the left lower parts of the specimen showed adenocarcinoma with lepidic pattern (magnification, $\times 10$ ).

average sample size was $3.65 \pm 1.27 \mathrm{~mm}$. Non-specificity was shown on 2 samples, and the qualified rate was $91.30 \%$ (21/23). There were 19 GGOs diagnosed by TBCB, with a diagnostic yield of $82.61 \%$ (19/23), of which 12 were adenocarcinoma, 5 were chronic inflammation, 1 was occupational interstitial lung disease, and 1 was a pulmonary meningothelial-like nodule. Of the 4 cases undiagnosed by TBCB, 2 were characterized as chronic inflammation by TBCB but turned out to be adenocarcinoma ( 1 indicated adenocarcinoma by cytology, and 1 was diagnosed by clinical and radiological manifestation), 2 provided unqualified samples, 1 was diagnosed as adenocarcinoma by clinical and radiological manifestation, the other presented nuclear atypia by a second biopsy (cytology and pathology), and was eventually diagnosed as adenocarcinoma based on clinical and radiological evidence (Table 1). Of the 16 cases finally recognized as adenocarcinomata, 12 underwent TBCB as well as cytology (brush and/or sheath wash), of which
5 were diagnosed by both of them, 3 were undiagnosed by either TBCB or cytology, 3 were diagnosed by ТВCB but not cytology, and 1 was identified by cytology but not TBCB. The diagnostic sensitivity of cytology was $50 \%$ (6/12), while it was $75 \%(12 / 16)$ for TBCB. There were 3 cases, whose final diagnosis were adenocarcinomas, that adopted forceps as well as TBCB simultaneously, of which 2 were identified by both of them, and 1 was diagnosed by TBCB but not forceps biopsy.

Univariate analysis was adopted to test multiple factors that may have affected the diagnostic yield. Although there is a tendency for large GGOs (GGOs $\geq 20 \mathrm{~mm}$ ) and fluoroscopically visible GGOs to have a higher diagnostic yield, the diagnostic yield was not altered significantly by any of the variables, including lesion size (GGOs $\geq 20 \mathrm{~mm}$ and GGOs $<20 \mathrm{~mm}$ ), navigation (VBN and $\mathrm{ENB}$ ), fluoroscopic visibility (visible and invisible), GGO component (pure GGOs and mixed GGOs), and type of GS 

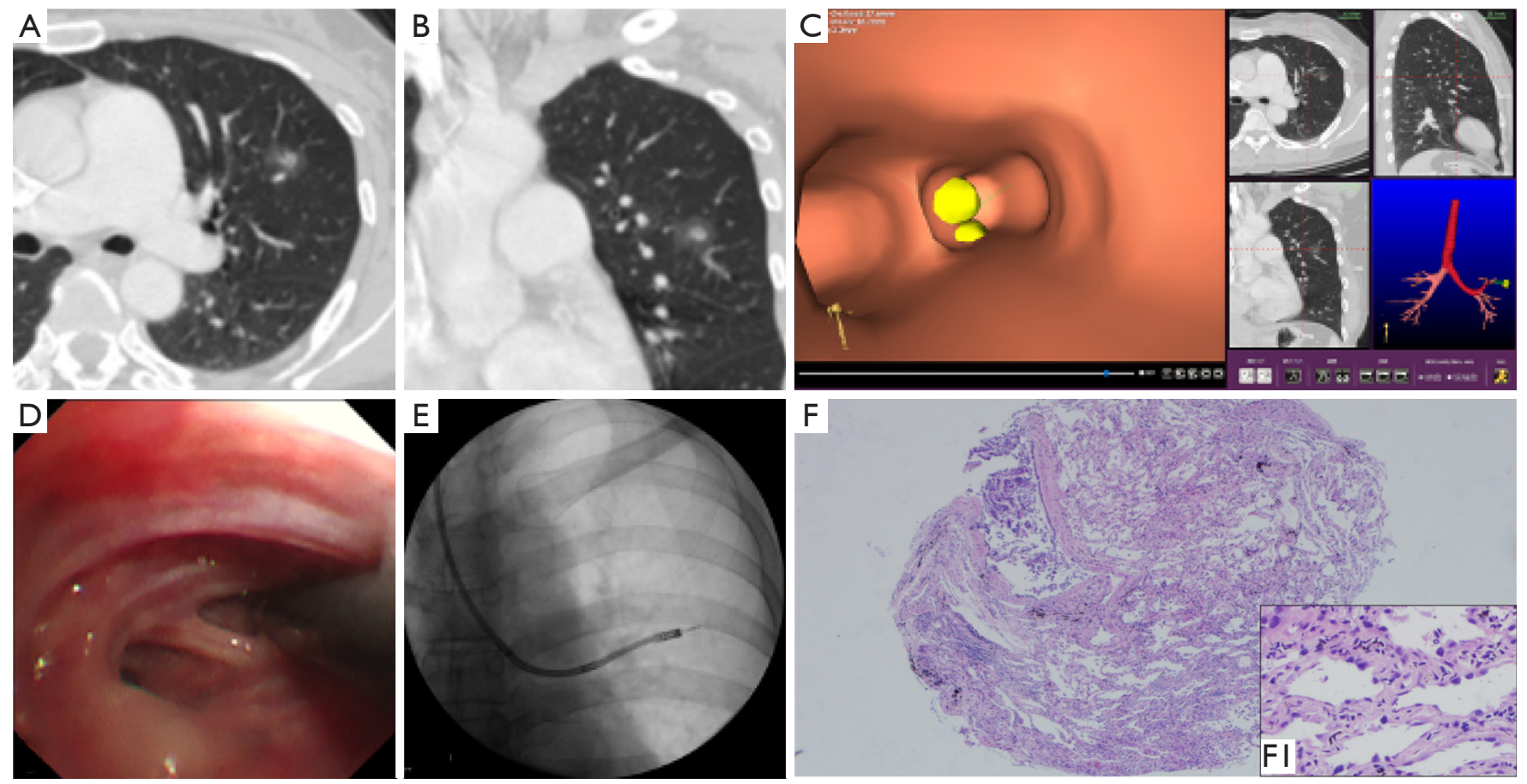

Figure 4 Representative case 3 of transbronchial cryobiopsy (TBCB) using the ultrathin bronchoscope (UTB) (BF-XP290) guided by virtual bronchoscopic navigation (VBN). (A) The high-resolution computed tomography (HRCT) axial section showed a pure ground-glass opacity (GGO) located in the anterior segment of the left upper lobe, with blood vessel involvement. (B) The HRCT coronal plane showed the GGO location. (C) Bronchial path and virtual bronchography of VBN. (D) The ultrathin cryoprobe was inserted into the target bronchus under the direct vision of the UTB. (E) Cryobiopsy was conducted under X-ray fluoroscopy. (F) Hematoxylin and eosin (H\&E) staining of the specimen with the magnification of $\times 4$. (F1) Enlarged picture displays atypical hyperplasia (magnification, $\times 20$ ). It was confirmed as adenocarcinoma in situ by surgery.

\section{(K-201 and K203) (Table 2).}

\section{Complications}

No pneumothorax or severe hemorrhage were presented in this study.

\section{Discussion}

It is very difficult to diagnose GGOs, as compared to solid nodules, by transbronchial lung biopsy (TBLB). Previous studies have reported that the diagnostic yield of TBLB with guided instruments for GGOs is $57-69 \%(8,9,11,20)$. We innovatively adopted TBCB using a $1.1 \mathrm{~mm}$ ultrathin cryoprobe, engaged with navigation bronchoscopy (VBN/ ENB), EBUS-GS and fluoroscopy, to achieve a diagnostic yield of $82.61 \%$. The majority of cases we enrolled was small solid nodules less than $30 \mathrm{~mm}$, and $60.87 \%(14 / 23)$ of them were less than $20 \mathrm{~mm}$; Of the 23 GGOs, 21 were
GGO dominated lesions, including 12 pure GGOs; All of them were risk factors for a poor diagnostic yield $(20,21)$. As a result, the powerful diagnostic efficacy of the ultrathin cryoprobe was prominently illustrated by the cases that otherwise were hard to operate and deemed a poor diagnostic yield by conventional TBLB.

Yarmus et al. conducted an animal study to evaluate the efficacy of the $1.1 \mathrm{~mm}$ ultrathin cryoprobe and found it provided comparable specimens with conventional TBCB in a comparable freezing period, of a much larger size than those obtained by TBLB (22). In this study, the

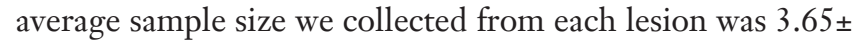
$1.27 \mathrm{~mm}$, with well-preserved cellular architecture, and almost devoid of crush artifacts. The large samples provided by TBCB are very helpful in making histologic diagnosis, especially for benign lesions and highly differentiated malignancies with minimal histologic heterogeneity, as they are capable of presenting a mix of pathology types (Figure 2G), along with normal tissues that contrast the 
Table 1 Baseline characteristics of patients with peripheral pulmonary ground-glass opacity lesions (GGOs)

\begin{tabular}{|c|c|}
\hline Characteristics & Value, mean $\pm S D$ \\
\hline Age (years) & $62 \pm 9.86$ \\
\hline \multicolumn{2}{|l|}{ Gender, No. } \\
\hline Male & $7(7 / 20)$ \\
\hline Female & $13(13 / 20)$ \\
\hline Lesion size (mm) & $21.58 \pm 11.88$ \\
\hline$\geq 20$ & $9(9 / 23)$ \\
\hline$<20$ & $14(14 / 23)$ \\
\hline \multicolumn{2}{|l|}{ Lobar location } \\
\hline RUL/LUL & $18(18 / 23)$ \\
\hline RML & $1(1 / 23)$ \\
\hline RLL/LLL & $4(4 / 23)$ \\
\hline \multicolumn{2}{|l|}{ GGO component } \\
\hline Pure GGO & $12(12 / 23)$ \\
\hline Mixed GGO & $11(11 / 23)$ \\
\hline $\mathrm{GGO} \geq 50 \%$ & $9(9 / 11)$ \\
\hline GGO <50\% & $2(2 / 11)$ \\
\hline \multicolumn{2}{|l|}{ GS } \\
\hline $\mathrm{K}-201$ & $13(13 / 22)$ \\
\hline $\mathrm{K}-203$ & $9(9 / 22)$ \\
\hline \multicolumn{2}{|l|}{ Bronchoscope } \\
\hline Standard bronchoscope & $9(9 / 22)$ \\
\hline Thin bronchoscope & $13(13 / 22)$ \\
\hline Ultrathin bronchoscope & $1(1 / 22)$ \\
\hline \multicolumn{2}{|l|}{ EBUS image } \\
\hline Blizzard sign & $13(13 / 22)$ \\
\hline Mixed blizzard sign & $9(9 / 22)$ \\
\hline \multicolumn{2}{|l|}{ X-ray fluoroscopy } \\
\hline Visible & $9(9 / 23)$ \\
\hline Invisible & $14(14 / 23)$ \\
\hline \multicolumn{2}{|l|}{ Navigation bronchoscopy } \\
\hline ENB & $9(9 / 23)$ \\
\hline
\end{tabular}

Table 1 (continued)
Table 1 (continued)

\begin{tabular}{lc}
\hline Characteristics & Value, mean \pm SD \\
\hline VBN & $14(14 / 23)$ \\
Final diagnosis & \\
Adenocarcinoma & $16(16 / 23)$ \\
Chronic inflammation & $5(5 / 23)$ \\
Occupational interstitial lung disease & $1(1 / 23)$ \\
Pulmonary meningothelial-like nodule & $1(1 / 23)$ \\
\hline
\end{tabular}

ENB, electromagnetic navigation bronchoscopy; EBUS, endobronchial ultrasound; GGO, ground-glass opacity; GS, guide sheath; LUL, left upper lobe; LLL, left lower lobe; RUL, right upper lobe; RML, right middle lobe; RLL, right lower lobe; VBN, virtual bronchoscopic navigation.

pathological area (Figure 3G). A previous study indicated that malignancy predicted a higher diagnostic yield (23). In this study, the diagnostic yield of benign GGOs was 100\% (7/7), and the diagnostic yield of malignant GGOs was $75 \%(12 / 16)$, the difference was not statistically significant. TBCB may be more competent in diagnosing GGOs than TBLB, since they usually suggests an early stage of malignancy with minimal changes in histology that not easy to be confirmed $(2,4)$. For example, there was 1 case that was identified as slightly atypical by TBLB, which was insufficient for a definitive diagnosis, but was diagnosed as a highly differentiated adenocarcinoma by TBCB.

Hemorrhage and pneumothorax are major complications of conventional cryoprobe and it has reportedly induced more bleeding than forceps $(14,24-26)$. We must stress that they are also considerable problems for the ultrathin cryoprobe. The specimen size obtained by the cryoprobe is increasing with increased freezing time, while larger specimen means severer damage to the lung tissue and more complications be indued. In this study, we set the freezing time at 3-5 s to make a balance between large samples and less complications. In large-scale application, however, an optimum freezing time is still in need to explore. Notably, unlike the conventional cryoprobe needed to be withdrawn along with the bronchoscope after biopsy and left the airway non-surveilled (27), the ultrathin cryoprobe can be retreated from the working channel of a standard 
Table 2 Diagnostic yield of transbronchial cryobiopsy and univariant analysis by different variables

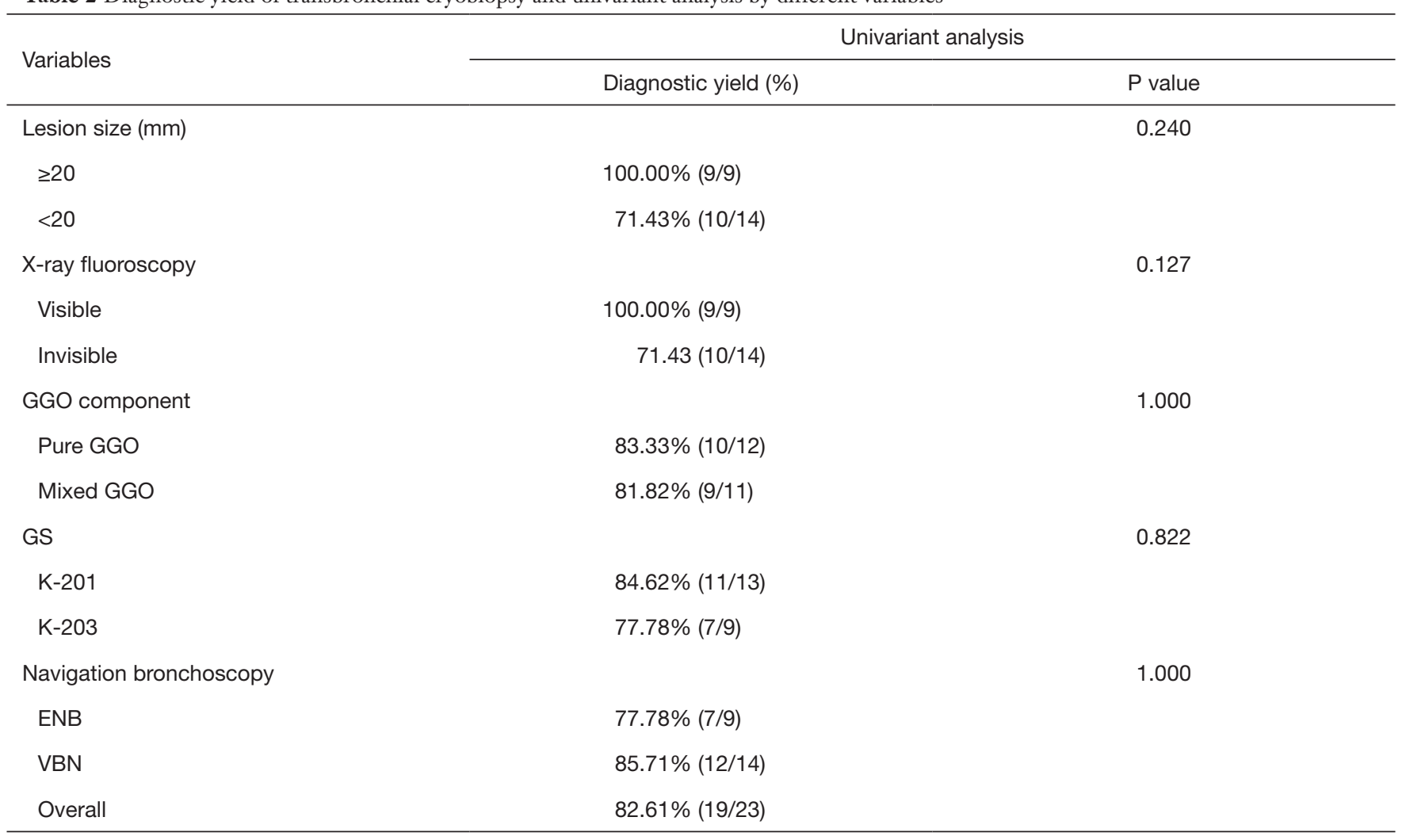

GGO, ground-glass opacity; GS, guide sheath; ENB, electromagnetic navigation bronchoscopy; VBN, virtual bronchoscopic navigation.

bronchoscope or a thin bronchoscope, so that the operator can earlier monitor and quickly handle the bleeding. What's more, the ultrathin cryoprobe can easily bend and extend to the distal bronchus, facilitating peripheral pulmonary biopsy. Remarkably, we adopted 3 kinds of modalities according to different situations as they presented. For a standard bronchoscope, the cryoprobe was small enough to be removed from the working channel of the K-203 GS, retaining the bronchoscope and GS in situ, so the GS could act as a locator to provide a simple and precise avenue for repetitive biopsy without requiring constant location verification by EBUS. The GS also blocked the airway and prevented potential bleeding; no additional insertions of a rigid bronchoscope or balloon catheter was needed. The thin bronchoscope was useful in dealing with remote targets, and although it required en bloc removal of the ultrathin cryoprobe and the K-201 GS, the bronchoscope remained on guard, which enabled a quick handling of potential complications. Additionally, there was 1 case in which we engaged the ultrathin cryoprobe with UTB under the guidance of VBN and fluoroscopy. The ultrathin cryoprobe is the first cryoprobe which can be incorporated with UTB. Both the UTB and the cryoprobe were very thin, and flexible enough to handle the complicated and sharp angles of the far distal left superior bronchus where the GGOs were located. The advantages offered by the combination of the UTB and cryoprobe allowed us to nearly conduct a direct vision biopsy.

In the univariant analysis, neither lesion size, fluoroscopic visibility, GGO component nor navigation system were identified as risk factors for a poor diagnostic yield. While GGOs $\geq 20 \mathrm{~mm}$ and fluoroscopically visible GGOs trend to yield higher than GGOs $<20 \mathrm{~mm}$ and fluoroscopically invisible GGOs. When compared the diagnostic yield of the ultrathin cryoprobe with previous records of TBLB for diagnosing GGOs $<20 \mathrm{~mm}$, the biopsy forceps yield 56.3-65.4\%, combined with guided bronchoscopy $(8,9,11,20)$, however, we yielded higher with $71.43 \%$. Fluoroscopically invisible GGOs often contribute to a lower diagnostic yield (20), because X-ray is unsubstituted for instructing biopsy instruments after EBUS was retreated (28). Meanwhile, GGOs beyond EBUS detection 
often frustrated in biopsies $(8,29)$. Navigation bronchoscopy, including VBN and ENB, facilitate confirmation of the targets by visualizing GGOs with image reconstruction and navigating bronchoscope to targeted bronchus, thus improving the diagnostic yield (9). In this study, $60.87 \%$ (14/23) of GGOs were fluoroscopically invisible, but with the help of navigation bronchoscopy, all GGOs, except 1 that did not adopt EBUS (21/22), were localized by EBUS. Moreover, we selectively adopted ENB for GGOpredominant lesions (GGO $\geq 50 \%$ ), and lesions that were difficult to operate on. There were 9 GGOs which adopted $\mathrm{ENB}$, of which 5 were fluoroscopically invisible, 8 were GGO-predominant; and the diagnostic yield was $77.78 \%$. A comprehensive use of navigation, EBUS, and fluoroscopy made up for the deficiency of using only 1 single technology.

Cytology examination, with a diagnostic sensitivity of $50 \%$, was not very effective for detecting malignancy in this study, because GGOs, that seldom infiltrate bronchus, made it difficult for malignant cells to be retrieved by the usual brushing and sheath wash. It is usual to adopt cytology after TBCB to improve its sensitivity, since TBCB penetrates the bronchus and destroys the integrity of GGOs, which makes the retrieval of cytological samples easier. Cytology is an essential complement for histology, as highlighted by 1 case that remained undiagnosed by TBCB as a cavitary lesion, yet presented as malignancy in brushing and cytopathology of sheath wash. Cavitary lesions with very little cellular structure were not easily localized, while brushing expanded the sampling area, and washing conducted after ТВСВ increased the probability of accurate lesion identification.

Our study involved some limitations. Firstly, this was a retrospective study with a limited sample size. Large-scale research is needed to further explore the diagnostic value and safety profile of the ultrathin cryoprobe. Secondly, bronchoscopes and sampling devices (e.g., cryoprobe, biopsy forceps, brush and sheath) were not fully randomized but applied based on the operator's individual experience, which made it difficult to confirm the diagnostic efficacy of each devices and their complementarity. Randomized tests are required to confirm whether the ultrathin cryoprobe is more efficient than forceps. Lastly, as a pilot study, we mainly adopted navigation bronchoscopy, EBUS and fluoroscopy for TBCB, but we also changed bronchoscopes, guide sheath and navigation according to different situations to explore the best combination. As a result, it is more difficult to deprive of the multiple confounding factors and find out the variables that had a definitive effect on the diagnostic yield. A large-scale, multi-center, randomized study should be arranged to explore the variables that altered the diagnostic yield.

\section{Conclusions}

We confirm that TBCB using the novel ultrathin cryoprobe can be engaged with a variety of bronchoscopes and guided instruments, to carry out peripheral pulmonary GGOs diagnosis. This is a feasible and safe approach, with a high diagnostic yield and few complications, especially for patients who are suspected to be at an early stage of pulmonary cancer.

\section{Acknowledgments}

Funding: This work was supported by National Key R\&D Program of China (2017YFC0112700), Shanghai Municipal Health and Medical Talents Training Program (2018BR09), Shanghai Municipal Education Commission-Gaofeng Clinical Medicine Grant Support (20181815).

\section{Footnote}

Reporting Checklist: The authors have completed the STRARD reporting checklist. Available at http://dx.doi. org/10.21037/tlcr-20-957

Data Sharing Statement: Available at http://dx.doi. org/10.21037/tlcr-20-957

Conflicts of Interest: All authors have completed the ICMJE uniform disclosure form (available at http://dx.doi. org/10.21037/tlcr-20-957). The authors have no conflicts of interest to declare.

Ethical Statement: The authors are accountable for all aspects of the work in ensuring that questions related to the accuracy or integrity of any part of the work are appropriately investigated and resolved. The protocol was approved by local ethics committee of Shanghai Chest Hospital (KS1829). All patients provided written informed consent before undergoing the procedure. All procedures performed in this study involving human participants were in accordance with the Declaration of Helsinki (as revised in 2013).

Open Access Statement: This is an Open Access article distributed in accordance with the Creative Commons 
Attribution-NonCommercial-NoDerivs 4.0 International License (CC BY-NC-ND 4.0), which permits the noncommercial replication and distribution of the article with the strict proviso that no changes or edits are made and the original work is properly cited (including links to both the formal publication through the relevant DOI and the license). See: https://creativecommons.org/licenses/by-nc-nd/4.0/.

\section{References}

1. Collins J, Stern EJ. Ground-glass opacity at CT: the ABCs. AJR Am J Roentgenol 1997;169:355-67.

2. Park CM, Goo JM, Lee HJ, et al. Nodular groundglass opacity at thin-section CT: histologic correlation and evaluation of change at follow-up. Radiographics 2007;27:391-408.

3. Engeler CE, Tashjian JH, Trenkner SW, et al. Groundglass opacity of the lung parenchyma: a guide to analysis with high-resolution CT. AJR Am J Roentgenol 1993;160:249-51.

4. Suzuki K, Kusumoto M, Watanabe S, et al. Radiologic classification of small adenocarcinoma of the lung: radiologic-pathologic correlation and its prognostic impact. Ann Thorac Surg 2006;81:413-9.

5. Callister ME, Baldwin DR, Akram AR, et al. British Thoracic Society guidelines for the investigation and management of pulmonary nodules. Thorax 2015;70 Suppl 2:ii1-ii54.

6. Rivera MP, Mehta AC, Wahidi MM. Establishing the diagnosis of lung cancer: Diagnosis and management of lung cancer, 3rd ed: American College of Chest Physicians evidence-based clinical practice guidelines. Chest 2013;143:e142S-e165S.

7. Tomiyama N, Yasuhara Y, Nakajima Y, et al. CTguided needle biopsy of lung lesions: a survey of severe complication based on 9783 biopsies in Japan. Eur J Radiol 2006;59:60-4.

8. Wang ZX, Li L, Zhang Z, et al. High-resolution computed tomography features and CT-guided microcoil localization of subcentimeter pulmonary ground-glass opacities: radiological processing prior to video-assisted thoracoscopic surgery. J Thorac Dis 2018;10:2676-84.

9. Huang $\mathrm{YH}$, Chen $\mathrm{KC}$, Chen JS. Ultrasound for intraoperative localization of lung nodules during thoracoscopic surgery. Ann Transl Med 2019;7:37.

10. Kobayashi Y, Ambrogio C, Mitsudomi T. Ground-glass nodules of the lung in never-smokers and smokers: clinical and genetic insights. Transl Lung Cancer Res
2018;7:487-97.

11. Ikezawa Y, Shinagawa N, Sukoh N, et al. Usefulness of Endobronchial Ultrasonography With a Guide Sheath and Virtual Bronchoscopic Navigation for Ground-Glass Opacity Lesions. Ann Thorac Surg 2017;103:470-5.

12. Schumann C, Hetzel J, Babiak AJ, et al. Cryoprobe biopsy increases the diagnostic yield in endobronchial tumor lesions. J Thorac Cardiovasc Surg 2010;140:417-21.

13. Nasu S, Okamoto N, Suzuki H, et al. Comparison of the Utilities of Cryobiopsy and Forceps Biopsy for Peripheral Lung Cancer. Anticancer Res 2019;39:5683-8.

14. Pajares V, Puzo C, Castillo D, et al. Diagnostic yield of transbronchial cryobiopsy in interstitial lung disease: a randomized trial. Respirology 2014;19:900-6.

15. Schuhmann M, Bostanci K, Bugalho A, et al. Endobronchial ultrasound-guided cryobiopsies in peripheral pulmonary lesions: a feasibility study. Eur Respir J 2014;43:233-9.

16. Xie F, Zhang J, Cao L, et al. Design of a prospective, multicenter, and cohort study of an innovative electromagnetic navigation bronchoscopy in diagnosing pulmonary nodules among Chinese population. J Thorac Dis 2019;11:5592-600.

17. Izumo T, Sasada S, Chavez C, et al. Radial endobronchial ultrasound images for ground-glass opacity pulmonary lesions. Eur Respir J 2015;45:1661-8.

18. Sasada S, Izumo T, Chavez C, et al. Blizzard Sign as a specific endobronchial ultrasound image for ground glass opacity: A case report. Respir Med Case Rep 2014;12:19-21.

19. Kurimoto N, Miyazawa T, Okimasa S, et al. Endobronchial ultrasonography using a guide sheath increases the ability to diagnose peripheral pulmonary lesions endoscopically. Chest 2004;126:959-65.

20. Ikezawa Y, Sukoh N, Shinagawa N, et al. Endobronchial ultrasonography with a guide sheath for pure or mixed ground-glass opacity lesions. Respiration 2014;88:137-43.

21. Okachi S, Imai N, Imaizumi K, et al. Factors Affecting the Diagnostic Yield of Transbronchial Biopsy Using Endobronchial Ultrasonography with a Guide Sheath in Peripheral Lung Cancer. Intern Med. 2016;55:1705-12.

22. Yarmus LB, Semaan RW, Arias SA, et al. A Randomized Controlled Trial of a Novel Sheath Cryoprobe for Bronchoscopic Lung Biopsy in a Porcine Model. Chest 2016;150:329-36.

23. Ali MS, Trick W, Mba BI, et al. Radial endobronchial ultrasound for the diagnosis of peripheral pulmonary lesions: A systematic review and meta-analysis. Respirology 
2017;22:443-53.

24. Ravaglia C, Wells AU, Tomassetti S, et al. Diagnostic yield and risk/benefit analysis of trans-bronchial lung cryobiopsy in diffuse parenchymal lung diseases: a large cohort of 699 patients. BMC Pulm Med 2019;19:16.

25. Kho SS, Chan SK, Yong MC, et al. Performance of transbronchial cryobiopsy in eccentrically and adjacently orientated radial endobronchial ultrasound lesions. ERJ Open Res 2019;5:00135-2019.

26. DiBardino DM, Haas AR, Lanfranco AR, et al. High Complication Rate after Introduction of Transbronchial Cryobiopsy into Clinical Practice at an Academic Medical Center. Ann Am Thorac Soc 2017;14:851-7.

Cite this article as: Jiang S, Liu X, Chen J, Ma H, Xie F, Sun J. A pilot study of the ultrathin cryoprobe in the diagnosis of peripheral pulmonary ground-glass opacity lesions. Transl Lung Cancer Res 2020;9(5):1963-1973. doi: 10.21037/tlcr-20-957
27. Guo S, Li Q, Jiang J, et al. Chinese expert consensus on the standardized procedure and technique of transbronchial cryobiopsy. J Thorac Dis 2019;11:4909-17.

28. Asano F, Ishida T, Shinagawa N, et al. Virtual bronchoscopic navigation without $\mathrm{X}$-ray fluoroscopy to diagnose peripheral pulmonary lesions: a randomized trial. BMC Pulmonary Medicine. 2017;17:184.

29. Yamada N, Yamazaki K, Kurimoto N, et al. Factors related to diagnostic yield of transbronchial biopsy using endobronchial ultrasonography with a guide sheath in small peripheral pulmonary lesions. Chest 2007;132:603-8.

(English Language Editor: J. Jones) 